Draft : Lamiroy B. \& Charolles M. (2011c). «Des formes aux sens : les clitiques dans la construction causative en faire". In G. Corminboeuf \& M-J. Béguelin eds. Du système linguistique aux actions langagières, De Boeck-Duculot, Louvain la Neuve, 41-59.

\title{
Des formes aux sens : les clitiques dans la construction causative en FAIRE
}

Béatrice Lamiroy

FRANITALCO, Université de Leuven

Michel Charolles

LATTICE, ENS Paris, Université de Paris-3

\section{Introduction}

Alain Berrendonner a manifesté tout au long de sa carrière la même sensibilité et le même respect pour la "grammaire des fautes" que le linguiste suisse Henri Frei, qui voyait dans les fautes un moyen de "prévenir ou de réparer les déficits du langage correct". Nous lui dédions cet article ${ }^{1}$ quil est consacré à des formes non canoniques attestées à l'oral (et que l'on trouve également sur Internet). Ces formes concernent la construction causative ${ }^{2}$ en faire et plus particulièrement le choix du clitique qui renvoie à ce que la grammaire traditionnelle appelait le "sujet" de l'infinitif, désigné comme "contrôleur" en grammaire générative depuis le modèle du Gouvernement et du Liage.

Dans les constructions causatives le clitique se met en principe à l'accusatif (1) ou au datif (2) selon que l'infinitif est un verbe intransitif ou transitif:

(1a) Max a fait rire les étudiants

(1b) Max les a fait rire

(2a) Max fait chanter une chanson à Léa

(2b) Max lui fait chanter une chanson

Tous les syntacticiens qui ont analysé la structure causative en français prévoient d'une façon ou d'une autre cette double règle dans leur système, qu'elle soit formulée en termes descriptifs d'alternance datif vs. accusatif (Blanche-Benveniste et al. 1987, Blanche-Benveniste 2007, Damourette \& Pichon 1911:§1105, Dannell 1979, Fournier 1998:112, Grevisse-Goosse 2008: $\S 903$, Herslund $1988: 63$ et 243, Le Goffic, $1993: 323$, Riegel $1996: 230$, Tasmowski 1985: 225 et 329), ou qu'elle corresponde à un mécanisme formel (transformationnel) dans des modèles tels que la grammaire générative (Kayne 1977: 196 ff, Roberts 1980, Rouveret \& Vergnaud 1980) ou la grammaire relationnelle (Comrie \& Polinsky 1993, Perlmutter 1983, Postal 1981, Fauconnier 1982). ${ }^{4}$

\footnotetext{
${ }^{1}$ Ce texte reprend et étoffe une version préalable présentée lors du Second Congrès Mondial de Linguistique Française (Lamiroy \& Charolles 2010)

2 La littérature consacrée à la construction causative étant énorme, nous nous limiterons ici à mentionner les sources pertinentes dans le cadre de notre article.

${ }^{3}$ Nous utilisons, à la suite de plusieurs auteurs (notamment Blanche-Benveniste 2007, Grevisse-Goosse 2008), les termes d'accusatif et de datif pour des raisons de commodité.

${ }^{4}$ Pour un aperçu détaillé de toutes ces analyses, voir Tasmowski (1985).
} 
Si la construction causative a retenu l'intérêt de tant de linguistes, c'est qu'elle est particulière du point de vue de la complémentation: contrairement à la structure infinitive à deux membres dont chacun peut avoir ses arguments comme en (3), la construction causative constitue un complexe verbal, ${ }^{5}$ c'est-à-dire un ensemble qui traite tous les arguments comme appartenant au verbe principal, comme le montre (4b), argument qui fait parfois traiter le verbe faire dans cette structure comme un auxiliaire diathétique (cf. Riegel 1996 :229):

(3a) Léa a prié Paul de répondre à la lettre

(3b) Léa l'a prié d'y répondre

(3c) *Léa l'y a prié de répondre

(4a) Léa a fait répondre Paul à la lettre

(4b) Léa l'y a fait répondre

(4c) ? Léa l' a fait $\mathbf{y}$ répondre ${ }^{6}$

Comme la construction causative, quelle qu'en soit la marque formelle, est attestée dans un très grand nombre de langues (Shibatani 1976, 2002), de nombreux linguistes se sont efforcés d'en fournir une définition sémantique universelle (e.a. Talmy 1976, Wierzbicka 1998, Wolff 2008). Du point de vue sémantique, les énoncés causatifs mettent toujours en jeu deux éléments au moins, une entité causatrice et une entité qui subit l'effet résultant de la causation (en anglais causee). L'entité causatrice est une force extérieure qui agit sur le "causee": c'est soit un agent, défini comme un être doué de volonté et capable de poursuivre un but, soit une force (aveugle). La situation causative est soumise à deux conditions essentielles : (a) l'évènement causé est temporellement postérieur à l'évènement causateur et (b) l'évènement causé est entièrement dépendant de l'occurrence de l'évènement causateur (Shibatani 2002). Toutefois, et c'est là sans doute un élément clé dans les données que nous allons traiter, le degré d'autonomie de l'entité qui subit la causation varie selon le cas et plusieurs types de causation peuvent être distingués en fonction du degré d'autonomie des acteurs en jeu et de la « directness » de la causation (Dixon 2000 : 67), c'est-à-dire selon que l'effet causé est obtenu directement ou indirectement. Dans les exemples suivants, ${ }^{7}$ on observe en effet que dans (5a) il y a un lien direct entre la cause (le professeur) et l'effet (réfléchir), tandis que dans (5b), la cause (New York) réalise de manière indirecte (par son architecture) l'effet obtenu (évoquer Hong Kong) :

(5a) Le professeur fournit aux élèves des outils d'analyse pour l'image animée ; il les fait réfléchir à la problématique de l'adaptation d'une oeuvre littéraire ...

(5b) Aujourd'hui les enfants nous disent que New York les fait penser à Hong Kong ! Mardi : Statue de la Liberté, Ellis Island

Entre ces deux extrêmes il existe un continuum causal (Tasmowski 1985:340, Shibatani 2002) allant du prototypiquement causatif à un effet affaibli de causation. Une des questions que nous poserons ici est de savoir s'il y a un lien entre le degré d'agentivité des sujets et la distribution des clitiques. Celle-ci, bien que la norme prévoie une règle qui consiste à alterner datif et accusatif selon le cas, s'avère en effet bien plus irrégulière qu'il n'y parait à première vue. C'est cette distribution irrégulière qui retiendra notre attention ici. Notons que des contre-exemples à la règle ont été relevés par la plupart des linguistes qui ont examiné la

\footnotetext{
${ }_{6}^{5}$ En grammaire relationnelle, ce phénomène est désigné par le terme d'union (cf. Perlmutter 1983).

${ }^{6}$ La phrase n'est pas impossible mais, comme le remarque Blanche-Benveniste (2007), faire est peu attesté dans la configuration où chaque verbe garde son autonomie. Selon Abeillé et al. (1996) en revanche, les deux configurations auraient le même statut.

${ }^{7}$ Tous nos exemples, sauf indication contraire, ont été trouvés sur Internet (Google).
} 
question. ${ }^{8}$ On trouve en effet le clitique datif avec des infinitifs intransitifs (6) et l'accusatif avec des verbes transitifs (7):

(6a) Si vous croyez que c'est commode de lui faire changer d'idée (P.Benoît cité GrevisseGoosse 2007: $§ 903)$

(6b) ... Un coup (...) qui l'a pourtant suffisamment effrayé pour lui faire renoncer à

son projet (A. Robbe-Grillet cité cité Grevisse-Goosse 2007:§903)

(6c) Elle lui fera téléphoner à ses parents (Kayne 1977:203, note 9)

(6d) Il est vrai que la forme de ce gros nuage lui fait penser à une silhouette de cheval ailé

(6e) Que Maman lui y fasse penser (Damourette \& Pichon 1911:\$1092, exemple oral)

(6f) Ça lui fera réfléchir / ça lui fera changer d'avis (Morin 1981)

(6g) Ça lui fait recourir à Marie (Tasmowski 1985:300)

D’après Grevisse-Goosse (2007: $§ 903)$, l'accusatif avec des infinitifs transitifs est surtout attesté dans des emplois plus anciens. Cependant les exemples ci-dessous montrent qu'on en trouve des exemples dans la langue actuelle, notamment dans les écrits de linguistes respectés:

(7a) Les vrais Juifs et les vrais chrétiens ont toujours attendu un Messie qui les ferait aimer Dieu (Pascal).

(7b) On ne la fera point dire ce qu'elle ne dit pas (Madame de Sévigné cité Grevisse-Goosse 2007:\$903)

(7c) Il m'est impossible de le faire aborder ce sujet (A. Duhamel cité Grevisse-Goosse 2007:\$903)

(7d) Il se sent fatigué, comme s'il venait de s'éveiller d'une nuit agitée et trop courte, et le frottement de ses pantoufles sur le plancher de bois nu le fait penser à du papier d'émeri (Paul Auster, Dans le scriptorium)

(7e) Le pénétrant parfum des lilas blancs la fit ouvrir la fenêtre (Damourette\&Pichon 1911:§1080, exemple de presse)

(7f) Ça la fait répondre des bêtises (Rouveret \& Vergnaud 1980)

(7g) Ça le faisait me répondre / me fuir (Tasmowski 1985:330)

Notons qu'on trouve parfois les deux clitiques chez le même auteur:

(8a) L'étonnement lui avait fait se dire tout bas ... (P. Bourget, cité Skårup 1985)

(8b) Cette admiration qui la faisait se dire ... (P. Bourget, ibidem)

ou avec le même infinitif, comme dans les exemples ci-dessous trouvés sur Internet:

(8c) Leur incarcération volontaire (...) les poussait à la mélancolie et leur faisait broyer du noir

(8d) Pour moi, les méthodes Ricci étaient trop violentes, elle déstabilisait les élèves, les faisait broyer du noir

Il est évident que l'utilisation de Google comme corpus demande des précautions, dans la mesure où on ne connaît pas l'identité des utilisateurs, qui peuvent en outre laisser des fautes de frappe. On peut néanmoins estimer qu'un locuteur qui utilise une structure aussi

\footnotetext{
${ }^{8}$ Il est intéressant de noter que les spécialistes de l'acquisition relèvent le même genre de "faute" chez de jeunes enfants, par ex. La fille lui fait rire (Bezinska et al. 2010).
} 
complexe que la causative en faire est quelqu'un qui manie le français avec une certaine compétence de la langue. Autrement dit, nous pensons que la confusion entre LE et LUI qu'on observe dans les données prises sur Internet mérite d'être signalée: ce que nous voulons faire ici n'est autre que d'attirer l'attention sur des cas attestés sur Google qui sont déviants par rapport à la norme traditionnelle (scolaire). Ils demanderaient à être soumis à une étude systématique et surtout à être complétés par une recherche sur un corpus oral soigneusement transcrit. Des sondages ponctuels sur Google montrent que la confusion va dans les deux sens, des infinitifs transitifs apparaissant avec l'accusatif, comme en (9) et des infinitifs intransitifs avec le datif, comme en (10): ${ }^{9}$

(9a) Mon fils de 7 mois et demi refusait le biberon du matin et du soir (...) et pour le soir plus de biberons je le fais manger un petit pot à base de laits et 2 petit suisses et tout se passe bien.

(9b) J'ai justement le livre "la cabane magique" : Panique à Pompéi, Le terrible empereur de Chine... Je le fais lire un chapitre quand il veut me faire plaisir

(9c) Une fois que j'ai suffisement d'or, je le fais construire un ziggourat, normalement, le tout ne prend pas plus de $2 \mathrm{~min}$

(9d) Donc je le fais acheter un câble ADSL blindé de $15 \mathrm{~m}$ de long. Et on réessaye ; ça marche, mais coupé toutes les 5 mn environ

(9e) Rédacteur en chef du journal «Le Matin », il la fait écrire quelques chroniques

(9f) Quand je veux couper les griffes, je couche mon chat, je le fais sentir la pince ${ }^{10}$

(10a) Puis, discrètement elle me dit que ça lui fait rigoler car elle adore qu'on soit brutal avec elle

(10b) Son mec est venu l'embrasser et lui gratifier d'un cunni qui lui fait mourir d'excitation

(10c) Si c'est vrai, c'est sûrement parce que ça leur fait déprimer d'en avoir une petite donc ils se rabattent sur la bouffe mais je pense que c'est une connerie

Nous nous intéresserons dans ce qui suit à l'alternance des deux clitiques illustrée ci-dessus. Afin de mieux comprendre leur distribution, nous nous sommes penchés sur un petit échantillon d'exemples qui se limitent aux infinitifs penser et réfléchir. Comme notre étude concerne les clitiques, elle ne prend pas en considération un certain nombre de structures apparentées:

- celle où l'objet de faire correspond à un SN lexical :

(11a) On a fait planter des choux aux linguistes (Herslund $1988: 238$ )

(11b) Je ferai partir John immédiatement (Kayne 1977 :196)

(11c) En lui donnant un coup de pied, elle a fait faire un tour à la boite de chocolats ${ }^{11}$

\footnotetext{
${ }^{9}$ Nous faisons abstraction ici de l'aspect quantitatif du problème qui demanderait évidemment à être pris en compte dans une étude systématique. Ainsi dans notre sondage, pour je le/la/les fais manger un/une ... nous avons obtenu un seul cas, alors que je lui/leur fais manger un/une ... donne 41 occurrences. Avec le verbe construire, les proportions étaient de 2 accusatifs vs 14 datifs.

10 Dans cet exemple, on a bien affaire au sens agentif de sentir 'renifler' et non pas au sens ergatif où le sujet a le rôle de thème, comme ce serait le cas dans Cette veste sent le tabac.

${ }^{11}$ Les cas où le référent de l'objet de faire est inanimé, comme dans cet exemple, sont rares pour des raisons évidentes : l'objet de la causation est un patient « responsable » (Damourette \& Pichon 1911, Herslund 1988), c'est-à-dire qu'il doit être doué d'une certaine autonomie pour pouvoir réaliser l'action indiquée par V-inf.
} 
- celle où le datif correspond non pas à l'agent mais au destinataire de l'infinitif (Herslund 1988 :243, Hulk 1984, Tasmowski 1983, Tayalati 2008) ; cette construction présente des contraintes particulières illustrées en $(12 \mathrm{~b})$ qui ne concernent pas le tour en cause ici :

(12a) On lui a fait parvenir le message

(12b) * On lui a fait téléphoner Marie

- celle où les clitiques correspondent à la 1re ou 2e personne ou au pronom réfléchi puisque l'opposition entre datif et accusatif y est neutralisée :

(13a) C'est alors que Jean Paulhan m'a fait avoir un prix prestigieux (R-J. Clot, cité Skårup 1985)

(13b) Fais-moi voir

(13c) Ils se sont fait sursauter mutuellement

- celle où l'agent est exprimé par un complément prépositionnel en par :

(14) On a fait planter les choux par les linguistes (Herslund 1988 :64)

Notons en passant que nous souscrivons à l'analyse de Herslund (1988 : 240) pour qui le datif fonctionne comme thème et sujet secondaire de la prédication ['on a fait : les linguistes ont planté des choux'], alors que la construction causative en par $S N$ est analysée comme une structure ergative puisque c'est l'accusatif qui est thème et sujet de la prédication secondaire passive ['on a fait : les choux ont été plantés par les linguistes']. ${ }^{12}$

Avant de passer à notre étude de cas (faire penser, faire réfléchir), nous évoquons brièvement les hypothèses qui ont été proposées dans la littérature pour rendre compte de la confusion entre le datif et l'accusatif à l'intérieur de la construction causative.

\section{Etat de la question}

Comme nous l'avons indiqué ci-dessus, la confusion qui s'observe quant à la distribution des deux clitiques a été remarquée par plus d'un linguiste. Dans la littérature, deux hypothèses en gros ont été proposées pour en rendre compte. L'une est d'ordre syntaxique (2.1.), l'autre propose en revanche une explication sémantique (2.2.).

2.1. Selon Dannell (1979 : 79), le datif apparaitrait avec un infinitif transitif indirect, là où on s'attendrait à un accusatif, quand le verbe admet dans sa valence à la fois un objet direct et un objet indirect. C'est le cas de verbes tels que croire, changer ou penser, par exemple: on trouve croire, penser à quelque chose mais aussi croire, penser quelque chose, changer de quelque chose mais aussi changer quelque chose. L'emploi du datif s'expliquerait alors par une contamination des deux constructions :

\footnotetext{
${ }^{12}$ Cette analyse rejoint en fait celle de Kayne (1977: 227), qui analyse la construction causative avec par SN à partir d'un V-inf passif. C'est bien la même intuition (on notera le passif soit opéré dans la paraphrase ci-dessous) qu'on retrouve chez Damourette \& Pichon (1936: § 2047), selon qui Le chirurgien fit opérer ce malade à son interne signifie que "la fin principale (...) c'est de faire que l'interne opère», tandis que dans Le chirurgien fit opérer ce malade par son interne « la fin principale (...) c'est que le malade soit opéré et par là débarrassé de son mal ».
} 
(15a) Mais toute tolérance accordée aux fanatiques leur fait croire immédiatement à de la sympathie pour leur cause (M.Yourcenar, cité Skårup 1985)

(15b) Un élu UMP déclare que Ségolène Royal lui fait penser à sa femme de ménage

(15c) Dans l'espoir de lui faire changer d'avis (Beja, cité Herslund 1988:246)

D'après le même auteur, la construction avec datif se serait progressivement étendue aux emplois avec un infinitif purement intransitif, comme rigoler dans les exemples ci-dessous que nous avons trouvés sur Internet. Bref, on aurait dans tous ces cas "un accusatif au niveau conceptuel mais un objet indirect au niveau syntaxique" :

(16a) Maman elle dit qu'ils vendent de la drogue (...) que c'est pour ça qu'ils tapent dans la main parce que la drogue, ça leur fait rigoler

(16b) Ils aiment bien se foutre la gueule d'eux-mêmes, ça leur fait rigoler

2.2. D'après Herslund (1990), il y aurait une généralisation du datif au détriment de l'accusatif pour des raisons sémantiques plutôt : avec le datif on assiste au "marquage de l'agent de V2 [= l'infinitif] pour bien souligner ce rôle d'agent et de sujet secondaire ». Cette hypothèse est séduisante dans la mesure où le datif est typologiquement reconnu (Lamiroy, 2000 ; Lazard, 1994 ; Van Belle et Van Langendonck, 1996-97) comme le cas le plus agentif après le nominatif : le datif correspond à quelqu'un "d'intéressé" par l'action, selon Damourette \& Pichon (1911:§1080), ou comme dit Herslund (1997), c'est le "deuxième à bord" dans la phrase, après le sujet. Notons pourtant que cette hypothèse est immmédiatement contredite par les cas où on trouve l'accusatif avec des infinitifs transitifs : dans ce dernier cas, l'objet de faire a nécessairement le rôle d'agent de V-inf (puisque l'infinitif a son propre patient), comme dans les exemples ci-dessous:

(17a) Des nouvelles un peu moins bonnes les firent précipiter leur départ (Gide, cité GrevisseGoosse 2007:§903)

(17b) Les femmes les plus naïves ont un sens merveilleux qui (...) les fait ressaisir bientôt tout l'empire qu'elles ont laissé perdre (Martin du Gard, cité Grevisse-Goosse 2007:\$903)

(17c) L'inquiétude naturelle aux malades qui les fait essayer sans cesse de nouveaux régimes (Bourget, cité Grevisse-Goosse 2007:§903)

Damourette \& Pichon (1911: §1105) qui avaient observé des cas qui n'obéissent pas à la norme, proposaient déjà une double analyse prévoyant une interprétation différente selon que la phrase se construit avec datif ou accusatif:

(18a) Je lui [fais chanter] une chanson

(18b) Je le fais [chanter une chanson]

Selon Damourette et Pichon, la construction où figure le complexe faire + V-inf "coalescent" s'impose si on a une chanson spécifique en tête (18a), tandis qu'on aura recours à (18b) si c'est l'activité de chanter une chanson qui importe, et non pas l'identité de la chanson.

Blanche-Benveniste (1978 : 195) formule également l'hypothèse que l'alternance datif vs. accusatif, là où elle se manifeste, est motivée, et en particulier par l'agentivité du sujet de faire. Ainsi, les deux clitiques seraient possibles chaque fois qu'une double lecture est possible. Toutefois, l'analyse de Blanche-Benveniste, contrairement à celle de Damourette \& Pichon, semble se concentrer sur l'agentivité du sujet principal plutôt que celle du sujet de l'infinitif. Dans (19a-b) par exemple, l'opposition correspondrait au fait que dans (19a) Paul 
fait en sorte qu'elle pense à son chimpanzé (qu'elle ne doit pas oublier de nourrir), alors que dans (19b) Paul a un physique qui rappelle celui d'un chimpanzé. Le sujet Paul est donc + agentif dans le premier cas (il est la cause directe de V-inf) et -agentif (il est la cause indirecte de V-inf) dans le deuxième, ce qu'on peut schématiser comme suit :

Lecture "A agentive" : "A faire X et X Cause B penser à son chimpanzé"

Lecture "A non agentive" : "A être X et X cause $\mathrm{B}$ penser à son chimpanzé"

Dans le deuxième cas, faire penser est quasiment lexicalisé, la phrase équivaut à 'il lui rappelle un chimpanzé' (par son physique) :

(19a) Paul la fait penser à son chimpanzé

(19b) Paul lui fait penser à son chimpanzé

Dans l'exemple suivant, l'accusatif serait préférable parce que le sujet principal est hautement agentif alors que celui de V-inf ne l'est aucunement, il n'est que patient de faire et prend par conséquent l'accusatif; (20b) serait douteux parce qu'un verbe statif comme ressembler est incompatible avec un "patient responsable » (Herslund 1988), rôle qui correspond au datif. Qu'il y ait un lien entre l'agentivité du sujet de l'infinitif et le choix du datif est également suggéré par le fait que les verbes ergatifs, dont le sujet grammatical ne porte jamais le rôle d'agent, semblent simplement exclure le datif (21b) :

(20a) Je la fais ressembler à sa soeur (dans le tableau que je suis en train de peindre)

(20b) ?? Je lui fais ressembler à sa soeur

(21a) Paul a fait tomber son frère / Paul l'a fait tomber

(21b) * Paul lui a fait tomber

C'est un contraste analogue qui expliquerait la différence qu'on observe entre $(22 a-b)::^{13}$ dans (22b), celui qui reconnait sa faute a été entièrement forcé de le faire (sous la torture par exemple), alors que dans (22a) on suggère qu'il a pris activement part au procès:

(22a) On lui a fait reconnaitre sa faute

(22b) ? On l'a fait reconnaitre sa faute

L'analyse de Abeillé et al. (1997: 66) rejoint celle esquissée ci-dessus. Selon ces auteurs, dans la paire

(23a) Je l'ai fait manger des épinards

(23b) Je lui ai fait manger des épinards

« l'individu dénoté par l' n'a pas eu d'autre choix que de manger les épinards, alors que la phrase contenant lui n'autorise pas cette inférence. » Mais Abeillé et al. notent également que les jugements d'acceptabilité concernant la structure causative varient fort selon les locuteurs. C'est ce que soulignent également Tasmowski \& Van Oevelen (1987 : 56) qui remarquent à propos des exemples (19a-b) de Blanche-Benveniste qu'il " existe indubitablement des variantes idiolectales ici. Pour nous $[=\mathrm{T} \& \mathrm{VO}]$, les gloses sont exactement inverses (nous soulignons) ». Selon les mêmes auteurs, de surcroît, " certains jugent que (a) mais non (b) possède les deux lectures. Ils interprètent alors (b) avec la glose de (a) ».

\footnotetext{
${ }^{13}$ Ces données nous ont été suggérées par Andrée Borillo (c.p., XIXe Colloque Lexique-Grammaire, Belgrade, septembre 2010).
} 
Autrement dit, aucune interprétation ne semble vraiment exclue et le moins qu'on puisse dire est que la signification de ce genre de phrases ne fait pas l'unanimité des locuteurs, même linguistes. Ou pour le dire encore autrement, la zone d'alternance entre datif / accusatif dans la structure causative en faire semble instable. Comme le suggèrent les exemples cidessus, un des facteurs qui rend l'analyse de la structure causative si difficile, et qui pourrait être à la base de la confusion des deux clitiques chez le locuteur, réside dans le fait qu'il y a toujours deux éléments potentiellement agentifs en jeu, et qu'ils peuvent l'être à des degrés divers selon le cas. C'est ce que suggèrent également les données que nous analysons ci-après (section 3) et c'est la raison pour laquelle nous invoquerons l'hypothèse du marquage différentiel de l'objet, phénomène bien connu des typologues (section 4).

\section{Etude de cas}

Les exemples suivants sont tous tirés de Google, ${ }^{14}$ ils contiennent soit penser à soit réfléchir $\grave{a}$. Nous avons ainsi examiné deux structures syntaxiques comparables: les deux verbes sélectionnent forcément un sujet + humain et sont intransitifs. Penser admet bien sûr également un complément d'objet direct dans sa valence, à l'encontre de réfléchir qui est toujours intransitif (transitif indirect dans la terminologie de la grammaire traditionnelle). Du point de vue quantitatif, penser semble bien plus courant après faire que réfléchir : une même consultation de Google fournit 1424 attestations de faire penser, contre 82 attestations de faire réfléchir. ${ }^{15}$

Quant à la distribution entre datif et accusatif, les deux verbes présentent des proportions inverses : alors que pour faire penser, $80 \%$ des exemples apparaissent avec le datif (lui/leur) contre $20 \%$ avec l'accusatif (le/la/les), $82 \%$ des phrases contenant faire réfléchir prennent l'accusatif et $18 \%$ seulement le datif. Mais on trouve des exemples de chaque cas pour chacun des verbes, penser (exemples en 24-25) comme réfléchir (exemples en 26-27) :

Datif:

(24a) Elle m'a dit que mon ami lui faisait penser à moi et que sa femme lui faisait penser à son père !

(24b) Les élèves revoient les éléments du poème de la création; ils choisissent et expliquent un élément qui leur fait penser à Dieu

(24c) Puis il se met ensuite à embrasser la pâte à modeler et me parler de son odeur qui lui fait penser à celle d'un bébé

Accusatif:

(25a) Il y a des gens qui disent que je les fais penser à Michel Serres. Je souris et je pense que ça vient des cheveux !

(25b) De même que le crépuscule le fait penser à Clélia, il peut aussi symboliser le point de changement dans la vie de Fabrice

(25c ) Le ruisseau d'or la fait penser à l'argent

\footnotetext{
${ }^{14}$ Il s'agit d'une consultation faite le 11/11/2009 à partir de la requête le/la/les fait penser à/aux et le/la/les fait réfléchir à/aux. Nous avons analysé 82 cas pour chacun des verbes.

${ }^{15}$ Une des raisons ici peut être la lexicalisation de faire penser qui signifie 'évoquer, rappeler': il n'y a pas de phénomène équivalent où faire réfléchir aurait un sens lexicalisé (figé).
} 
Datif:

(26a) Lors de cet entretien, la directrice évalue le profil de l'étudiant, lui fait réfléchir aux défis à relever et propose les solutions de suivi

(26b) La Vertu de Prudence est une qualité qui dirige l'homme vers le Bien et lui fait réfléchir aux moyens d' y arriver

(26c) Aussi, il leur fait réfléchir à d'autres solutions pour envisager un développement durable, tout en satisfaisant à leurs besoins

Accusatif:

(27a) Elle sensibilise les enfants et les fait réfléchir à leurs habitudes de consommation

(27b) Vu leur âge c'est uniquement la situation qui les fait réfléchir à leur passé

(27c) Cette visite les fait réfléchir à d'autres possibilités d'orientation que les lycées généraux

La prépondérance de datifs avec penser (alors que l'accusatif est dominant dans le cas de réfléchir) plaide en faveur de l'hypothèse syntaxique de Dannell (1979), puisque penser a une double valence, admettant soit un objet direct soit un objet indirect. On notera toutefois que, comme l'a remarqué Skårup (1990) en parlant d'un exemple de Herslund, ${ }^{16}$ ces cas avec datif sont syntaxiquement étonnants dans la mesure où la phrase contient deux compléments indirects pour un seul verbe, complexe il est vrai : en principe un verbe n'est jamais accompagné de deux objets indirects à la fois. Comme l'illustrent les exemples ci-dessus, de manière flagrante, le caractère animé ou non du sujet principal ne semble pas jouer de rôle décisif dans la distribution du datif/accusatif, ni dans le cas de penser ni dans celui de réfléchir d'ailleurs : quand le sujet de faire est animé, on observe le datif (penser: ex. 24a, réfléchir: 26a-26c) tout comme l'accusatif (penser: ex. 25a, réfléchir: 27a). Il en va de même lorsque le sujet est inanimé: le clitique est soit un datif (penser: ex. 24b-c, réfléchir: 26b), soit un accusatif (penser : ex. 25b-25c, réfléchir : ex. 27b-27c). Nos données brouillent donc non seulement les pistes concernant la distribution datif vs accusatif, mais semblent remettre également en question l'hypothèse, très plausible à première vue, selon laquelle la causation directe serait plutôt associée à des SN de type + animé, la causation indirecte aux SN de type -animé (Verhagen \& Kemmer 1997): la réflexion des enfants, due à cette visite en (27c), ne semble pas a priori causée de manière plus indirecte que celle due à elle dans (27a).

Même si tous les objets de faire sont nécessairement de type + humain dans nos exemples, puisque les deux infinitifs penser et réfléchir sélectionnent par définition un sujet humain, force est d'admettre qu'il existe une différence importante entre les deux: avec réfléchir, le sujet est nécessairement agentif, il est activement impliqué par sa pensée tandis qu'avec penser, le degré d'agentivité varie selon le cas, comme l'avait bien remarqué Blanche-Benveniste au sujet des exemples (19a-b). Dans la première série d'exemples cidessous (28a-d), le sujet de l'infinitif est bien l'agent de la pensée, alors que dans la deuxième série 529a-d), il n'en est que le siège : dans ces cas, faire penser est proche du sens 'évoquer, rappeler' et la causation est indirecte, passant souvent par le biais d'une image. On a donc affaire à une lexicalisation de faire penser, analogue à celle qu'on observe dans faire comprendre, faire voir, etc. Ce qui est pourtant crucial, c'est que le degré d'agentivité de celui qui pense ne semble pas être un critère absolument décisif pour le choix du clitique puisqu'on trouve avec faire penser aussi bien le datif que l'accusatif, comme le montrent encore les exemples suivants. Dans la première série (28a-d), il est difficile de remplacer faire penser par 'rappeler, évoquer' et la lecture préférentielle est donc bien celle où le sujet de penser est

\footnotetext{
${ }^{16}$ L'exemple dont il s'agit est "la luminosité des feux arrière lui fit penser à une voiture allemande".
} 
agentif, tandis que dans la seconde (29a-d), faire penser peut être lu comme un synonyme de 'rappeler, évoquer'. Ce qui est frappant c'est qu'on observe dans les deux séries aussi bien le datif que l'accusatif:

(28a) Il se trouve que celle-ci a très peu d'amis et se retrouve donc souvent seule ce qui la fait penser à son passé

(28b) Aussi quand le petit prince fait de la montagne, il fait une expérience qui le fait penser aux gens. Au sommet de la montagne il écoute l'écho et il trouve ...

(28c) Car la confrontation d'un enfant à la mort lui fait penser à sa propre mort, sans doute pour la première fois

(28d) L'enseignante leur fait penser aux documentaires qu'ils ont vus dans leur passé. Etaient-ils équitables? Auraient-ils dû l'être ?

(29a) Parce que la lumière des éoliennes la fait penser à un incendie

(29b) Pour la petite histoire, mes frangines me disent que cette photo les fait penser à

Wilma Petersen, ou Gil Grissom du feuilleton télé « Les Experts »

(29c) Les hommes sont si attirés par les seins des femmes car ça les fait retourner en enfance, ça leur fait penser au sein de leur mère

(29d) Depuis quelque temps il lui dit qu'elle lui fait penser à la Gestapo ! Trop c'est trop pour Marie

Dans certains cas d'ailleurs, les phrases sont indéterminées entre une lecture agentive et non agentive du sujet de l'infinitif, comme dans les exemples en (30) où on trouve également les deux clitiques :

(30a) Après avoir fait le ménage dans sa vie, voilà qu'elle s'attaque à ce qui lui fait penser à son ex

(30b) La mer évoque l'éveil de la nature, dans un paysage maritime, l'éveil de l'espoir dans un pays étranger qui le fait penser à sa patrie

Il est évident que de plus amples recherches, tant du point de vue de la taille des corpus que du choix des infinitifs se combinant avec faire sont indispensables pour étayer, ou infirmer, les observations présentées ci-dessus. Dans l'ensemble, pour les cas observés ici, l'instabilité entre le datif et l'accusatif semble en tout cas nettement plus importante que ne le laissent entendre les grammaires : nos exemples montrent clairement, nous semble-t-il, que la règle retenue tant par la grammaire traditionnelle que par les spécialistes des grammaires formelles correspond à une tendance plus qu'à une véritable règle. C'est la raison pour laquelle il est intéressant de renvoyer ici à certains travaux en typologie qui ont montré, tout à fait indépendamment de la construction causative, que la zone de l'objet direct à référent humain est naturellement instable, et que celui-ci est soumis dans beaucoup de langues à ce que les typologues appellent un "marquage différentiel".

\section{Le phénomène du marquage différentiel de l'objet}

Le phénomène du marquage différentiel de l'objet ("differential object marking", abrégé DOM dans les travaux de typologie), attesté dans un grand nombre de langues ${ }^{17}$ consiste dans le fait que la marque formelle qui caractérise l'objet direct diffère selon le référent auquel il renvoie. Dans ces langues, l'objet direct est marqué d'une façon particulière quand il s'agit

\footnotetext{
${ }^{17}$ Environ 300 langues, incluant des langues de familles génétiquement indépendantes tels le hindi, le roumain ou le macédonien par exemple.
} 
d'un référent qui a plus de chances "d'être un sujet qu'un objet". Lorsque une entité prédestinée à être sujet fontionne (quand même) comme objet dans la phrase, elle reçoit une marque spéciale, soit casuelle soit par un accord entre le verbe et l'objet (Bossong 1991). ${ }^{18} \mathrm{La}$ base cognitive de ce phénomène correspondrait au fait que les locuteurs catégorisent les entités en associant leurs traits sémantiques référentiels à des fonctions syntaxiques privilégiées, notamment en fonction de leur probabilité d'apparaitre comme sujet de la phrase (Aissen 2003). On sait depuis Li (1976) que le sujet prototypique présente universellement deux traits, à savoir [+ agentif] et [+ topique]. Les entités qui se caractérisent par une grande probabilité d'apparaitre comme sujet dans la phrase correspondront par conséquent à des $\mathrm{N}$ qui sont hautement agentifs et hautement topicaux: dans les langues à DOM, ces entités, ressenties comme présentant des affinités naturelles avec la fonction sujet, reçoivent une marque spéciale lorsqu'elles remplissent une fonction autre que celle de sujet, en l'occurrence celle (qui ne leur est pas naturelle) d'objet. Quant à la question de savoir quelles entités sont ressenties par les locuteurs comme hautement agentives et topicales, on sait que si toutes les langues ne traitent pas les choses de la même façon, elles semblent tout de même respecter une hiérarchie universelle d'agentivité (Givon 1976, Silverstein 1976) et de topicalité (Du Bois 2003) qu'on peut représenter comme suit :

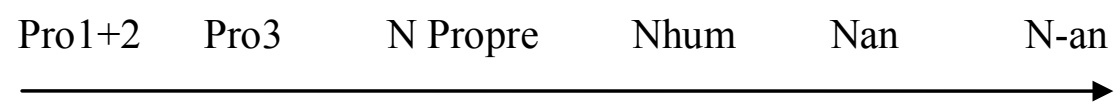

Ce qui est important pour notre propos est que les pronoms personnels (le Pro1, c'est-à-dire "je", serait ainsi le topique par excellence) se situent plus haut sur l'échelle que les SN lexicaux : ${ }^{19}$ nous y reviendrons ci-dessous.

Le phénomène du DOM n'affecte pas le français, mais est attesté dans une langue qui est génétiquement proche du français, à savoir l'espagnol. En espagnol, l'objet direct connait deux types de marquage selon que le $\mathrm{N}$ est $[ \pm$ hum] et/ou [ \pm spécifique]: en effet on introduit la préposition A devant l'objet « direct » quand le référent est [+hum] et [+spéc], ${ }^{20}$ auquel cas on parle d'un « accusatif prépositionnel », ce qui est en fait une contradiction dans les termes, puisque la préposition A marque normalement, en espagnol comme en français, le complément d'objet "indirect". Le clitique correspondant à "l'accusatif prépositionnel" est bien sûr le datif le, et non l'accusatif lo, phénomène bien connu de l'espagnol et décrit comme "leismo". L'objet direct dont le référent est [+hum] est formellement marqué comme un objet indirect, parce que celui-ci est plus proche, sémantiquement, du sujet: l'objet indirect porte le plus souvent le trait [+hum] et il est plus topical que l'objet direct (Lazard 1994, Herslund 1988). ${ }^{21}$ Ce qu'il importe de noter ici est qu'il y a dans des langues à DOM comme l'espagnol, une neutralisation manifeste entre l'objet direct et l'objet indirect (31a), ou entre l'accusatif et le datif pour ce qui est des clitiques (31b), puisque le datif s'utilise pour marquer ce qui est fonctionnellement un objet direct:

\footnotetext{
18 "Some entities are more likely to function as subjects than as objects. When they are used as objects, they are MARKED in a special way» (Bossong 1991).

${ }^{19}$ De même, un SN défini est plus haut sur l'échelle de l'agentivité/topicalité qu'un SN -défini + spécifique, qui l'est à son tour par rapport à un SN -spécifique. Un SN singulier est également plus haut placé dans cette même hiérarchie qu'un SN pluriel (Du Bois 2003).

${ }^{20}$ En fait la distribution de l'accusatif dit prépositionnel en espagnol est une question bien plus complexe qu'on ne le dit ici, et son extension a évolué au cours de l'histoire et varie selon la région (Melis et Flores 2009).

${ }^{21}$ Ce serait la raison pour laquelle le complément d'objet indirect apparait le plus souvent dans les corpus sous la forme d'un pronom, celui-ci se plaçant plus haut dans la phrase qu'un SN lexical (ce qu'ont remarqué Herslund 1988 pour le français, et Du Bois 2003 pour l'anglais).
} 
(31a) Vi a Juan en el cine je-vis à Jean dans le cinéma J'ai vu Jean au cinéma
$\begin{array}{lll}\text { Le } & \text { vi } & \text { en el cine } \\ \text { PRO-DAT:3SG } & \text { je-vis } & \text { dans le cinéma }\end{array}$
'Je l'ai vu au cinéma' (litt. Je lui ai vu au cinéma)

Il est intéressant de signaler qu'on assiste à la neutralisation inverse dans certaines variantes de l'espagnol où le clitique accusatif est utilisé à la place du datif pour marquer l'objet indirect, comme dans

(32) (A Maria) la dije que era guapa

(A Marie) PRO-ACC:3SG je-dis que elle-était belle

'(A Marie) je lui ai dit qu'elle était belle' (litt. A Marie ${ }_{i}$ je $l_{i}$ 'ai dit qu'elle était belle)

Ce dernier phénomène, appelé "loismo" ou "laismo" (selon que le référent de l'objet est masculin ou féminin), confirme - et c'est ce qu'on retient de tout ce qui précède - qu'il existe une zone où naturellement accusatif et datif se confondent, celui où on a affaire à un $\mathrm{SN}$ à référent humain qui remplit la fonction syntaxique d'objet. Cette situation rappelle évidemment le cas de figure que nous avons décrit plus haut concernant la construction causative: bien que le français ne soit pas une langue à DOM comme l'espagnol, on peut formuler l'hypothèse que l'objet direct de faire dans la construction causative subisse le même effet que l'objet direct tout court en espagnol. La confusion du locuteur qui doit choisir entre le datif et l'accusatif dans la structure causative serait par conséquent bien plus naturelle qu'il n'y parait à première vue. Il existe évidemment une différence typologique importante entre le français et l'espagnol: alors que l'espagnol marque également le SN lexical comme un datif quand il remplit la fonction d'objet direct (cf. ex. 31a), le français ne neutralise le datif et l'accusatif qu'au niveau du clitique (et non pas à celui des SN lexicaux):

a. Je lui fais penser à sa mère

b. Je le fais penser à sa mère

c. Je fais penser Jean à sa mère

d. * Je fais penser à Jean à sa mère

Mais précisément, l'on sait que les langues n'ont pas toutes le même "cut-off point» sur l'échelle hiérarchique relative à l'agentivité/topicalité des $\mathrm{N}$ indiquée ci-dessus: certaines langues incluent par exemple les N+animés dans les cas à DOM alors que d'autres s'arrêtent "avant", aux N+humains (Melis \& Flores 2005). Ici on observe que si l'espagnol étend le DOM jusque dans la zone des SN lexicaux, le français le limite à la zone des clitiques seulement, à l'exclusion des SN lexicaux (et dans la structure causative seulement).

\section{Conclusions}

Nous avons montré dans cet article dédié à Alain Berrendonner que les formes syntaxiques sont naturellement complexes - et en tout cas plus que ne le laisse entendre la grammaire normative traditionnelle - lorsque le sens est complexe, la structure causative mettant en jeu à la fois, du point de vue syntaxique, deux propositions imbriquées l'une dans l'autre, et au 
niveau sémantique, de multiples acteurs susceptibles d'agir à des degrés divers sur la situation que la causation va entraîner ou modifier.

Si les propriétés syntaxiques de l'infinitif qui suit faire jouent certainement un rôle dans l'alternance des clitiques, la complexité sémantique de la structure causative y est sans doute également pour beaucoup. En effet, comme toute causation met en cause au moins deux acteurs, chacun avec une agentivité relative, on conçoit que le continuum causatif comprenne une série de cas de figure se situant dans une zone intermédiaire où l'opposition entre accusatif et datif se neutralise et qui fait que le locuteur fasse appel tant à l'un qu'à l'autre de ces deux formes. Du point de vue référentiel, on peut en effet analyser une situation dans laquelle $\mathrm{A}$ fait que $\mathrm{B}$ mange sa soupe de plusieurs manières :

\begin{tabular}{|l|l|l|l|l|}
\hline & & A & B \\
\hline & & Agentif & Agentif & Volitionnel \\
\hline 1 & Je le (lui) fais manger sa soupe & + & - & - \\
\hline 2 & Je le (lui) fais manger sa soupe & + & - & + \\
\hline 3 & Je lui (le) fais manger sa soupe & + & + & - \\
\hline 4 & Je lui fais manger sa soupe & + & + & + \\
\hline
\end{tabular}

$1:$ B n'est pas en mesure physiquement de manger sa soupe et il n'en a pas la volonté (les grands malades, les bébés)

$2: B$ voudrait manger mais ne peut pas manger sa soupe sans une aide

3 : B peut la manger mais ne le veut pas

4 : je fais simplement penser à B qu'il devrait manger sa soupe parce qu'il a faim (les enfants qui peuvent manger seuls, qui ont faim, mais qui ne pensent pas à manger)

Le datif est possible dans tous les cas mais on l'attendrait plus à partir 3 et même de 2 , et il serait quasiment obligatoire dans 4. On comprend aisément qu'indépendamment des considérations syntaxiques, l'accusatif sera d'autant plus facilement " confondu » avec le datif que l'agent de V-inf porte le trait + humain, comme c'est le cas avec les verbes penser et réfléchir examinés ici.

Nous avons essayé de montrer par ailleurs que si les locuteurs confondent souvent (en tout cas dans une langue proche de l'oral comme celle utilisée sur Internet) les deux cas dans la structure causative avec faire, cela n'est après tout pas si étonnant si on pense que des centaines de langues au monde neutralisent systématiquement la différence entre le datif et l'accusatif lorsque certaines conditions - celles qui sont précisément caractéristiques de l'objet direct de faire - sont réunies.

\section{Références}

Abeillé, A., Godard, D. \& Miller, P. (1996). Les causatives en français: un cas de compétition syntaxique. Langue française, 115, 1, 62-74.

Aissen, J. (2003). Differential object marking: Iconicity vs. Economy. Natural Language and Linguistic Theory, 21, 435-483.

Bezinska Y, Chevrot J-P., Novakova I, Nardy A. (2010). L'acquisition de faire + Vinf en français: production, compréhension, imitation. Deuxième Congrès Mondial de Linguistique française, Nouvelle Orléans, juillet 2010. 
Blanche-Benveniste, C., Deulofeu, J., Stefanini, J. et Van Den Eynde, K. (1987). Pronom et syntaxe: l'approche pronominale et son application au français. Paris. SELAF.

Blanche-Benveniste, C. (2007). Les énoncés à causatifs réfléchis. In: Rousseau, A., Bottinau, D. et Roulland, D. (éds). L'énoncé réfléchi. Rennes: Presses Universitaires de Rennes, 155-173.

Bossong, G. (1991). Differential Object marking in Romance and beyond. In Wanner, D. et Kibbee, D. (éds.) New Analyses in Romance Linguistics. Amsterdam: J. Benjamins, 143-170.

Comrie, B. et Polinsky, C. éds (1993). Causatives and Transitivity. Amsterdam: J. Benjamins.

Dannell, K.J. (1979). Remarques sur la construction dite causative. Faire (laisser, voir, entendre, sentir) + Infinitif. Stockholm: Almqvist \& Wiksell.

Dixon, R.M.W. (2000). A typology of causatives: form, syntax and meaning. In Dixon, .M.W et Aikhenvald, A.(éds) Changing valency. Cambridge: CUP, 30-82.

Du Bois, W. (2003). Argument structure: Grammar in use. In: Du Bois, W., Kumpf, L.E. \& Ashby, W.J. (éds). Preferred argument structure. Grammar as architecture for function. Amsterdam: J. Benjamins, 11-60.

Fauconnier, G. 1982. Generalized Union. In: Tasmowski, L. \& Willems, D. (éds) Problems in Syntax. Gent: Communication and Cognition, 195-230.

Frei, Henri (1929). La grammaire des fautes. Rééd. 2004. Genève: Ennoïa.

Fournier, N. (1998). Grammaire du français classique, Paris, Belin.

Givon, T. (1976). Topic, pronoun and grammatical agreement. In: Li, C. (éd.), 149-188.

Grevisse-Goosse, A. (2008). Le Bon Usage. Bruxelles: De Boeck-Duculot.

Herslund, M. (1988). Le datif en français. Louvain: Peeters.

Herslund, M. (1990). Remarques sur la construction causative: Réponse à P. Skårup. Aarhus Universitet: Romansk Institut, (Pré)publications.

Hulk A. (1984). De la syntaxe du pronom en dans la construction quantitative. Revue Québecoise de linguistique 3, 167-199.

Kayne, R. (1977). Syntaxe du français. Paris: Seuil.

Lamiroy, B. (2000). De la syntaxe comparée des langues romanes: du passif au datif possessif. In: Schösler, L. (éd.) Le passif. Etudes romanes, n ${ }^{\circ}$ spécial 45, 135-154.

Lamiroy, B. \& Charolles, M.(2010) Les clitiques accusatifs versus Datifs dans les constructions cuasatives en faire. Deuxième Congrès Mondial de Linguistique française, Nouvelle Orléans, juillet 2010.

Lazard, G. (1994). L'actance. Paris: PUF.

Le Goffic, P. (1993). Grammaire de la phrase française. Paris : Hachette.

Li, C. (éd.) (1976). Subject and topic. New York: Academic Press.

Melis, Chantal \& Flores, Marcela. (2009). On the interplay on the forces of erosion and forces of repair in language change. Folia Linguistica Historica, 30, 1-40.

Morin, Y.-C. (1980). Les bases syntaxiques des règles de projection sémantique: l'interprétation des constructions en 'faire'. Linguisticae Investigationes, 4, 203-212.

Perlmutter, D. (1983). Studies in Relational Grammar, vol. I. Chicago: Chicago University Press.

Postal, P. (1981). A failed analysis of the French cohesive infinitive construction. Linguistic Analysis, 8, 281-324.

Riegel, M., Pellat, J.-C. et Rioul, R. (1996). Grammaire méthodique du français. Paris : PUF.

Rouveret, A. et Vergnaud, J.R. (1980). Specifying Reference to the Subject: French causatives and Conditons on Representations. Linguistic Inquiry, 11, 97-202.

Roberts, I. (1980). French Causatives in generative syntax. Ph.D. Georgetown University, ms.

Shibatani, M. (1976). The grammar of causative constructions. In: Shibatani, M. (ed.) Syntax and Semantics 6. New York: Academic Press, 1-42. 
Shibatani, M. (2002). The causative continuum. In: Shibatani, M. (ed.) The Grammar of Causation and Interpersonal Manipulation. Amsterdam: J. Benjamins, 136-177.

Silverstein, M. (1976). Hierarchy of features and ergativity. In: Dixon, R.M.W. (éd.) Grammatical categories in Australian languages. Canberra: Australian Institute of Aboriginal Studies, 112-171.

Skårup, P. (1985). Compte-rendu de Dannell 1979.

Skårup, P. (1990). Faire + Infinitif selon Michael Herslund. Aarhus Universitet: Romansk Institut, (Pré)publications.

Tasmowski-De Ryck L. (1984). ?*Lui faire téléphoner quelqu'un d'autre : une stratégie ?, Lingvisticae Investigationes VIII : 2, 403-427.

Tasmowski-De Ryck L. (1985). Faire infinitif. In : L. Melis et al. (eds), Les constructions de la phrase française. Gent: Communication et Cognition, 233-365.

Tasmowski-De Ryck L. et Van Oevelen, H. (1987). Le causatif pronominal. Revue Romane, 22, 40-58.

Tayalati, F. (2008). La distinction ergatif/inergatif et son incidence sur le placement des clitiques datifs dans les constructions causatives avec faire et rendre. Probus, 20-2, 301-321.

Van Belle, W. and W. Van Langendonck éds. (1996-1997). The Dative, 2 Volumes. Amsterdam/Philadelphia: John Benjamins.

Verhagen, A. \& Kemmer, S. (1997). Interaction and causation: Causative constructions in modern standard Dutch. Journal of Pragmatics, 27, 1, 61-82. 